\title{
Parameter Evaluation Procedure for Gapped Core Current Transformers Considering Accuracy Requirements under Transient Conditions
}

\author{
Victor Vorobiev ${ }^{1}$, Vadim Moskalenko ${ }^{1}$, Anton Rasshcheplyaev ${ }^{1}$, Goda Nudelman $^{2}$, Alexander Navolochny $^{2}$, Olga \\ Onisova $^{3, *}$, and Ivan Naumov ${ }^{3}$ \\ ${ }^{1}$ System Operator of the United Power System, Relay Protection and Automation Department, Moscow, Russia \\ ${ }^{2}$ All-Russian Scientific, Research, Designing and Technological Institute of Relay Construction with Pilot Production, Cheboksary, \\ Russia \\ ${ }^{3}$ Chuvash State University named after I.N. Ulianov, Faculty of Energy and Electrical Engineering, Cheboksary, Russia
}

\begin{abstract}
Particular features of gapped core current transformers and characteristics of transient phenomena inside them are under consideration; a procedure is proposed that allows for evaluating the excitation limiting e.m.f. and overall dimensions of the CT required for installation, based on known parameters of the transient conditions in CT installation point and the specified CT error.
\end{abstract}

\section{Introduction}

Nowadays, electromagnetic current transformers (CTs) with closed core are used, as a rule, to connect the relay protection circuits in power system of Russia. Modern CTs are manufactured of electrical steel characterized by the hysteresis loop similar to rectangle and, thereby, high values of residual flux of the CT magnetic core (up to $86 \%$ ) [1]. Combination of the high value of CT residual flux and presence of decaying dc component of the corresponding polarity in short circuit current can result in fast CT saturation under transient condition and incorrect operation of relay protection devices connected to the CT [2]. In order to limit the residual magnetic flux of magnetic core, CTs can be manufactured with air gaps in the core (accuracy classes of CTs: PR, TPY, TPZ). Application of gapped core CTs allows for avoiding the CT saturation due to high values of remanent flux of the CT magnetic core, but requires consideration of the particular features of phenomena in their secondary circuits, change in the approach to select the CT parameters.

\section{Excitation Characteristic of Gapped Core CTs}

In any operation conditions of $\mathrm{CT}$, magnetizing current adjusted to one turn, can be calculated using the expression [3]:

$$
i_{0}=H_{\mathrm{ST}} l_{\mathrm{ST}}+H_{\mathrm{G}} l_{\mathrm{G}},
$$

where $H_{\mathrm{ST}}$ and $H_{\mathrm{G}}$ - field intensity in steel and gap, respectively; $l_{\mathrm{ST}}$ and $l_{\mathrm{G}}$ - length of steel part of magnetic core and width (length) of air gap, respectively.
Field intensity in steel is related to magnetic induction determined by the dependence $H_{\mathrm{ST}}=f(B)$; field intensity in the gap can be determined using the expression $H_{\mathrm{G}}=B / \mu_{0}$, where $\mu_{0}=4 \pi \cdot 10^{-7} \mathrm{H} / \mathrm{m}$ - air magnetic permeability. Taking into account (1) the dependence of specific magnetizing current from the induction in magnetic circuit with the gap:

$$
i_{\text {OSpec }}=\frac{i_{0}}{l_{\mathrm{ST}}}=f(B)+B N_{\mathrm{p}},
$$

where $N_{\mathrm{p}}=\frac{l_{\mathrm{G}}}{\mu_{0} l_{\mathrm{ST}}}-$ demagnetization factor of magnetic circuit.

Figure 1 shows the influence of the air gap relative length $l_{\mathrm{G}} / l_{\mathrm{ST}}$ on the type of the $\mathrm{CT}$ excitation characteristic. The CT minimum gap is selected due to the reduction of residual magnetic flux up to considerably low values, and for high quality cold-rolled steels it is about 0.001; but under the terms for ensuring the operation accuracy of CTs under transient conditions, the design gap shall be selected several times higher than the minimum.

\section{Equivalent Circuit for Gapped Core CTs}

The expression (1) can be presented as follows:

$$
i_{0}=\frac{B l_{\mathrm{ST}}}{\mu_{\mathrm{ST}}}+\frac{B l_{\mathrm{G}}}{\mu_{0}}=B Q\left(\frac{1}{\lambda_{\mathrm{ST}}}+\frac{1}{\lambda_{\mathrm{G}}}\right)=\frac{B Q}{\lambda_{0}},
$$

where $\mu_{\mathrm{ST}}-$ absolute magnetic permeability of the magnetic circuit steel, $Q-$ its cross-section area, $\lambda_{\mathrm{ST}}-$ magnetic conductivity of steel, $\lambda_{\mathrm{G}}-$ magnetic

\footnotetext{
* Corresponding author: onisova-vniir@ya.ru
} 
conductivity of air gap, $\lambda_{0}=\frac{\lambda_{\mathrm{ST}} \lambda_{\mathrm{G}}}{\lambda_{\mathrm{ST}}+\lambda_{\mathrm{G}}}-$ magnetic conductivity of the whole magnetic core.

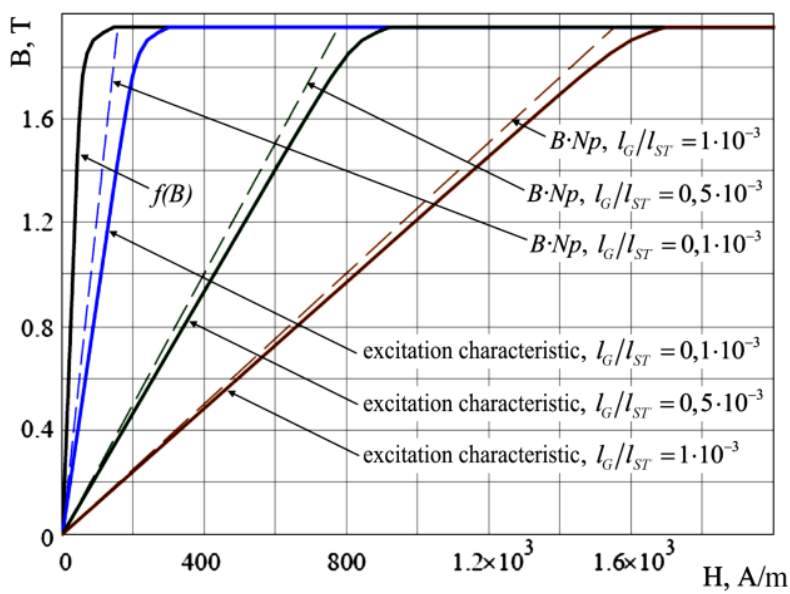

Fig. 1. Influence of the Air Gap Relative Length on the CT Excitation Characteristic

Inductances of the electrical circuit adjusted to one turn $L_{\mathrm{ST}}, L_{\mathrm{G}}, L_{0}$, equal by value, conform to magnetic conductances $\lambda_{\mathrm{ST}}, \lambda_{\mathrm{G}}, \lambda_{0}$. Therewith, in compliance with the expression (2) in equivalent circuit for transformer with a gap in magnetic core, common excitation branch can be replaced with two parallel branches (Figure 2), corresponding to the inductance of steel (non-linear) and the inductance of air gap (linear). As a rule, the current flowing in the branch from $L_{\mathrm{G}}$ is considerably higher than the current, flowing in the branch from $L_{\mathrm{ST}}$ (as $L_{\mathrm{G}}<L_{\mathrm{ST}}$ ); due to this linearization of CT magnetization curve is achieved.

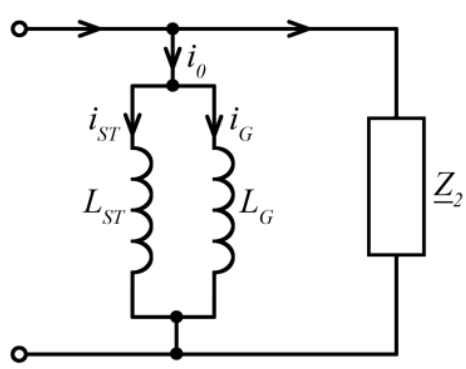

Fig. 2. Equivalent Circuit for CTs with Nonmagnetic Gaps ( $\underline{Z_{2}}$ - total resistance of the secondary circuit)

\section{Secondary Loop Time Constant of Gapped Core CT}

Secondary loop time constant $T_{S}$ is calculated by the ratio of total inductance of the secondary loop to its total active resistance. At absolutely active burden of CT $T_{S}=L_{0} / R_{2 \Sigma}$.

According to the equivalent circuit for gapped core CTs, presence of nonmagnetic gaps in the magnetic core leads to reduction in the secondary loop inductance and, consequently, to reduction of the secondary loop time constant.
Inductance of steel magnetic core without gap adjusted to one turn, can be determined as follows:

$$
L_{\mathrm{ST}}=\lambda_{\mathrm{ST}}=\frac{Q \mu_{\mathrm{ST}}}{l_{\mathrm{ST}}} .
$$

Inductance of the magnetic core with nonmagnetic gap adjusted to one turn:

$$
L_{0}=\lambda_{0}=\frac{\lambda_{\mathrm{ST}} \lambda_{\mathrm{G}}}{\lambda_{\mathrm{ST}}+\lambda_{\mathrm{G}}}=\frac{Q \mu_{\mathrm{ST}} \mu_{\mathrm{G}}}{\mu_{\mathrm{ST}} l_{\mathrm{G}}+\mu_{\mathrm{G}} l_{\mathrm{ST}}} .
$$

Ratio of inductances $L_{0}$ and $L_{\mathrm{ST}}$ shows, what fold the secondary loop time constant changes depending on the length of nonmagnetic gap in magnetic circuit. As per (3) and (4):

$$
\frac{L_{0}}{L_{\mathrm{ST}}}=\frac{Q \mu_{\mathrm{ST}} \mu_{\mathrm{G}} /\left(\mu_{\mathrm{ST}} l_{\mathrm{G}}+\mu_{\mathrm{G}} l_{\mathrm{ST}}\right)}{Q \mu_{\mathrm{ST}} / l_{\mathrm{ST}}}=\frac{1}{\mu_{\mathrm{ST}} * \frac{l_{\mathrm{G}}}{l_{\mathrm{ST}}}+1},
$$

where $\mu_{\mathrm{ST}^{*}}=\mu_{\mathrm{ST}} / \mu_{\mathrm{G}}=\mu_{\mathrm{ST}} / \mu_{0}-$ relative magnetic permeability of steel.

\section{Accuracy Classes of Gapped Core CTs [4]}

By the nature of imposed requirements to accuracy of gapped core CTs, the following CT accuracy classes are divided:

- PR - characteristics of transient conditions are not rated: the requirements are imposed to CT accuracy at effective value of short circuit current;

- TPY - characteristics of transient conditions are rated: the requirements are imposed to instantaneous value of CT error during short circuit transient conditions;

- TPZ - characteristics of transient conditions are rated: the requirements are imposed to periodic component of CT error during short circuit transient conditions.

\section{Parameter Evaluation Procedure for Gapped Core CTs}

A set of CT parameters, including excitation limiting e.m.f., secondary loop time constant, overall dimensions, can be calculated more precisely by the CT manufacturer only, based on the parameters of the primary network and requirements to $\mathrm{CT}$ accuracy class provided to him. Nevertheless, at the stage of design works, it is preferably to have an idea about values of all or several magnitudes specified, in order to select one or another class of $\mathrm{CT}$, to carry out preliminary (comparative) feasibility study of the variants.

The engineering practice given below allows for preliminary evaluation of excitation limiting e.m.f., time constant of the secondary circuit and (if overall dimensions require evaluation) the cross-section and the length of the magnetic path of the CT to be installed.

CT parameters are evaluated in the following sequence:

1) Initial (start) value of design secondary loop time constant is taken equal to eternity: $T_{s . \text { des }}^{0}=\infty$. 
2) Dependence of the transient factor [4] on the time $K_{\mathrm{tf}}^{0}(t)$ is calculated, and maximum value of transient factor $K_{\text {tf.max }}^{0}$ for $T_{s \text {.des }}^{0}=\infty$ is determined.

Transient factor at short circuit without automatic reclosing cycle is calculated using the expression [4]:

$$
K_{\mathrm{tf}}(t)=\frac{\omega T_{p} T_{s}}{T_{p}-T_{s}}\left(e^{-\frac{t}{T_{p}}}-e^{-\frac{t}{T_{s}}}\right)-\sin (\omega t),
$$

where $\omega$ - circular frequency of industrial current, $T_{p}-$ primary time constant.

In the cycle of non-successful reclosing "Close Open - Close", the transient factor is determined as follows. In the course of the first short circuit $\left(0 \leq t \leq t_{\text {closel }}\right) \quad K_{\mathrm{tf}}(t)$ changes in accordance with expression (5). After fault clearance, during reclosing dead time $\left(\mathrm{t}_{\text {closel }}<t \leq\left(\mathrm{t}_{\text {closel }}+\mathrm{t}_{\text {open }}\right)\right) K_{\text {tf }}(t)$ attenuates at exponential rate with the secondary loop time constant, starting from the value which it had at the moment of first short circuit clearance $\left(t_{\text {closel }}\right)$ :

$$
K_{\mathrm{tf}}(t)=K_{\mathrm{tf}}\left(t_{\text {closel }}\right) e^{-\frac{t-t_{\text {closel }}}{T_{s}}} .
$$

In the course of the second short circuit $\left(t>\left(t_{\text {closel }}+t_{\text {open }}\right)\right)$, the change $K_{\mathrm{tf}}(t)$ can be expressed as follows:

$$
K_{\mathrm{tf}}(t)=K_{\mathrm{tf}}\left(t_{2}\right)+\frac{\omega T_{p} T_{s}}{T_{p}-T_{s}}\left(e^{-\frac{t-t_{2}}{T_{p}}}-e^{-\frac{t-t_{2}}{T_{s}}}\right)-\sin \left(\omega\left(t-t_{2}\right)\right),
$$

where $t_{2}=t_{\text {closel }}+t_{\text {open }}-$ duration of the first short circuit and dead time.

Maximum value of the transient factor $K_{\text {tf.max }}$ is determined as maximum value $K_{\mathrm{tf}}(t)$ for the time of transient phenomenon under consideration.

3) Value of the secondary loop time constant is clarified:

$$
T_{s . \mathrm{des}}^{1}=\frac{K_{\mathrm{tf} \max }^{0}}{\omega \varepsilon_{\max }} .
$$

Calculation of the secondary loop time constant and transient factor provides iteration procedure*: after execution of step 3, repeat the step 2, calculating $K_{\text {tf.max }}^{1}$ taking into account the clarified value $T_{s . \text { des }}^{1}$, then again step 3 (calculate $T_{s . \text { des }}^{2}$ ), etc. Calculations shall be completed when the difference between the calculated value $K_{\text {tf.max }}^{n}$ and the value obtained during the previous iteration $K_{\text {tf.max }}^{n-1}$ does not exceed the accepted value of accuracy $\varepsilon_{\text {it \% }}$ :

\footnotetext{
${ }^{*}$ If the amplitude value of error periodic component $\varepsilon$ $\varepsilon_{a c \max }$ is set under transient condition (CT of TPZ class), design value of the secondary loop time constant shall be determined using the expression $T_{s \text {.des }}=1 /\left(\omega \varepsilon_{a c \max }\right)$; then the transient factor shall be calculated. Iteration procedure of calculation in this case is not required.
}

$$
\frac{K_{\mathrm{tf} \text { max }}^{n}-K_{\mathrm{tf.max}}^{n-1}}{K_{\mathrm{tf} \text { max }}^{n}} \times 100 \leq \varepsilon_{\mathrm{it} \%} .
$$

Value of the secondary loop time constant obtained upon completion of iteration calculations shall comply with the requirements of the CT phase angle [4]:

$$
\Delta \varphi[\min ] \geq \frac{3438}{\omega T_{s . \operatorname{des}}^{n}[\mathrm{~s}]} .
$$

4) Initial value of the CT secondary winding resistance is taken equal to zero: $R_{2 \text { des }}^{0}=0 \Omega$.

5) Design excitation limiting e.m.f. $E_{\text {al. des }}^{0}$ is determined for $R_{2 . \text { des }}^{0}=0 \Omega[4]$ :

$$
E_{\text {al. des }}=\frac{K_{\text {ssc.act }} K_{\text {tf.max }}}{1-K_{r}} I_{2 . \text { rat }}\left(\mathrm{R}_{2}+\mathrm{R}_{\text {b.act }}\right),
$$

where $K_{\text {sscact }}$ - actual value of the periodic component short circuit current factor in design condition, $K_{\text {ssc }}=I_{1 \text {.short circuit }} / I_{1 \text {.rat }} ; I_{1 \text {.short circuit }}-$ effective value of periodic component of short circuit current in design condition in the point of installation of the $\mathrm{CT}$ under consideration; $I_{1 . \text { rat }}$ - rated primary current of CT; $K_{r}-$ remanence factor; $I_{2 \text { rat }}$ - rated secondary current of CT; $\mathrm{R}_{2}-$ DC resistance of the secondary winding; $\mathrm{R}_{\text {b.act }}-$ actual active resistance of the CT secondary circuit burden.

6) The required cross-section area of CT is evaluated using the expression:

$$
Q_{\text {des }}^{0}=\frac{E_{\text {al. des }}^{0}}{\omega w_{2 . \text { des }} B_{\text {exc. des }} / \sqrt{2}},
$$

where $w_{2 \text { des }}-$ design number of turns of the CT secondary winding; $B_{\text {exc. des }}-$ value of magnetic induction of steel saturation (for modern grades of electrical cold-rolled anisotropic steel the following can be accepted: $B_{\text {exc. des }} \approx 1.8-1.9 \mathrm{~T}$ ).

7) The value of the CT secondary winding resistance is clarified. For this purpose, the formula can be used to take into account the expected number of turns of secondary winding and the value of CT cross-section area calculated above:

$$
R_{2 . \text { des }}^{1}=\frac{\rho \cdot 2 \sqrt{\pi Q} \cdot w_{2}}{S_{\text {cond }}},
$$

where $S_{\text {cond }}-$ cross-section area of the secondary winding conductors; $\rho-$ specific resistivity of the conductor material.

Calculation of the excitation limiting e.m.f. and the cross-section area of CT also provides iteration procedure: after clarification of the secondary winding resistance $R_{2 \text {.des }}^{1}$ (step 7), repeat the calculation (clarify) the excitation limiting e.m.f. $E_{\text {al. des }}^{1}$ (step 5) and the cross-section area $Q_{\text {des }}^{1}$ (step 6); calculations shall be repeated until the difference between the calculated value in the actual iteration $Q_{\text {des }}^{n}$ and the value obtained during the previous iteration $Q_{\text {des }}^{n-1}$ exceeds the accepted value of accuracy $\varepsilon_{\text {it } \%}$ : 


$$
\frac{Q_{\mathrm{des}}^{n}-Q_{\mathrm{des}}^{n-1}}{Q_{\mathrm{des}}^{n}} \times 100 \leq \varepsilon_{\mathrm{it} \%} .
$$

8) The length of nonmagnetic gap $l_{\text {G.des }}$, at which design value of the CT secondary loop time constant is ensured, shall be evaluated:

$$
l_{\text {g.des }}=\frac{Q \mu_{0} w_{2 . \text { des }}^{2}}{L_{0}}-\frac{l_{\mathrm{ST}} \mu_{0}}{\mu_{\mathrm{ST}}},
$$

where $L_{0}=T_{s . \text { des }}\left(R_{2 \text {.des }}+R_{\text {1.act }}\right)$ - inductance of excitation branch.

Considering the fact that CT excitation branch excitation is determined predominantly by the nonmagnetic gap parameters, length of the gap can be calculated using the simplified expression:

$$
l_{\text {g.des }}=\frac{Q \mu_{0} w_{2 . \text { des }}^{2}}{L_{0}} .
$$

Relative value of the nonmagnetic gap length (and the length itself) shall comply with the condition of restriction of residual flux of the $\mathrm{CT}$ magnetic circuit:

$$
l_{\text {g.des* }}=\frac{l_{\text {g.des }}}{l_{\mathrm{ST}}} \geq \frac{\mu_{0}}{\mu_{\mathrm{ST}}}\left(\frac{K_{r . \mathrm{ST}}}{K_{r}}-1\right) .
$$

where $K_{r . \mathrm{ST}}-$ remanence factor of CT with closed magnetic core.

\section{Examples of CT Parameters Calculation}

Initial data for calculation of parameters for gapped core CTs are specified in Table 1. Calculations are made for two values of the primary network time constant: 30 and $100 \mathrm{~ms}$. CTs of TPY and TPZ classes are examined. Amplitude value of total error in transient mode $\left(\varepsilon_{\max }\right)$ for CT of TPY class is taken equal to $10 \%$, amplitude value of periodic component of error $\left(\varepsilon_{a c \text { max }}\right)$ for CT of TPZ class is $5.2 \%$.
Results of CT parameters calculation, carried out as per the procedure given above, are specified in Table 2 . Diagrams for variation of the CT instantaneous error under transient condition, illustrating the particular features of behavior of CT classes under examination, are shown in Figures 3 and 4.

In case of relatively large nonmagnetic gap (CT of TPZ class), a considerable fraction of current d.c. component is absorbed by the CT excitation branch and is not converted to the secondary circuit; therewith, a probability of CT saturation in transient mode reduces, but instantaneous error of CT sufficiently increases (see Figure 3).

Length of nonmagnetic gap according to which the secondary circuit time constant is determined, also has a considerable influence on the CT behavior during the reclosing dead time. In case of small lengths of nonmagnetic gap (CT of TPY class), CT instantaneous error cannot fall to zero during the dead time (CT fails to demagnetize); consequently, during the second cycle of short circuit the error takes sufficiently higher values than during the first cycle (see Figure 4). In case of large air gap, instantaneous error reduces almost to zero during the dead time (CT is practically fully demagnetized), therewith, during the second cycle of short circuit the CT accuracy almost does not increase compared to the first cycle (see Figure 3 ).

The results obtained confirm the practicability to use CTs of TPZ class at quite high values of time constant of the primary network (80-100 ms and more): under any equal conditions the excitation voltage and overall dimensions of TPZ class CT, for which conversion of short circuit current periodic component is rated only, can be considerably low, than TPY class CT, which requires precise conversion of total short circuit current (requirements are imposed to total error of transient condition).

\begin{tabular}{|c|c|c|c|c|c|c|c|}
\hline Parameter & $E_{\text {al.des }}, \mathrm{kV}$ & $Q, \times 10^{-4}, \mathrm{~m}^{2}$ & $l_{\mathrm{ST}}, \mathrm{m}$ & $l_{\mathrm{G}}, \mathrm{mm}$ & $R_{2}, \Omega$ & $T_{s}, \mathrm{~ms}$ & $K_{r}$, p.u. \\
\hline \multirow{8}{*}{ Value } & \multicolumn{7}{|c|}{$\mathbf{T P Z}, T_{p}=30 \mathrm{~ms}$} \\
\hline & 530 & 6.108 & 1.2 & 5.489 & 3.986 & 61.2 & 0.02 \\
\hline & \multicolumn{7}{|c|}{$\mathbf{T P Z}, T_{p}=100 \mathrm{~ms}$} \\
\hline & 1070 & 12.4 & 1.2 & 9.455 & 5.666 & 61.2 & 0.02 \\
\hline & \multicolumn{7}{|c|}{$\mathbf{T P Y}, T_{p}=30 \mathrm{~ms}$} \\
\hline & 1368 & 15.757 & 1.2 & 1.918 & 6.406 & 344 & 0.1 \\
\hline & \multicolumn{7}{|c|}{$\mathbf{T P Y}, T_{p}=100 \mathrm{~ms}$} \\
\hline & 6868 & 79.681 & 1.2 & 1.919 & 14.392 & 1014 & 0.1 \\
\hline
\end{tabular}

Table 1. Initial Data for CT Parameters Calculation

\begin{tabular}{|c|c|c|c|c|c|c|c|c|}
\hline Parameter & $I_{1 . \text { rat }}, \mathrm{A}$ & $I_{2 . \text { rat }}, \mathrm{A}$ & $R_{\text {b.act }}, \Omega$ & $\begin{array}{c}K_{\text {ssc.act }}, \\
\text { p.u. }\end{array}$ & $T_{p}, \mathrm{~ms}$ & $t_{\text {closel }}, \mathrm{ms}$ & $t_{\text {close2 }}, \mathrm{ms}$ & $t_{\text {open }}, \mathrm{ms}$ \\
\hline Value & 2,000 & 1 & 5 & 10 & 30,100 & 100 & 100 & 400 \\
\hline
\end{tabular}

Table 2. Results of CT Parameters Calculation 


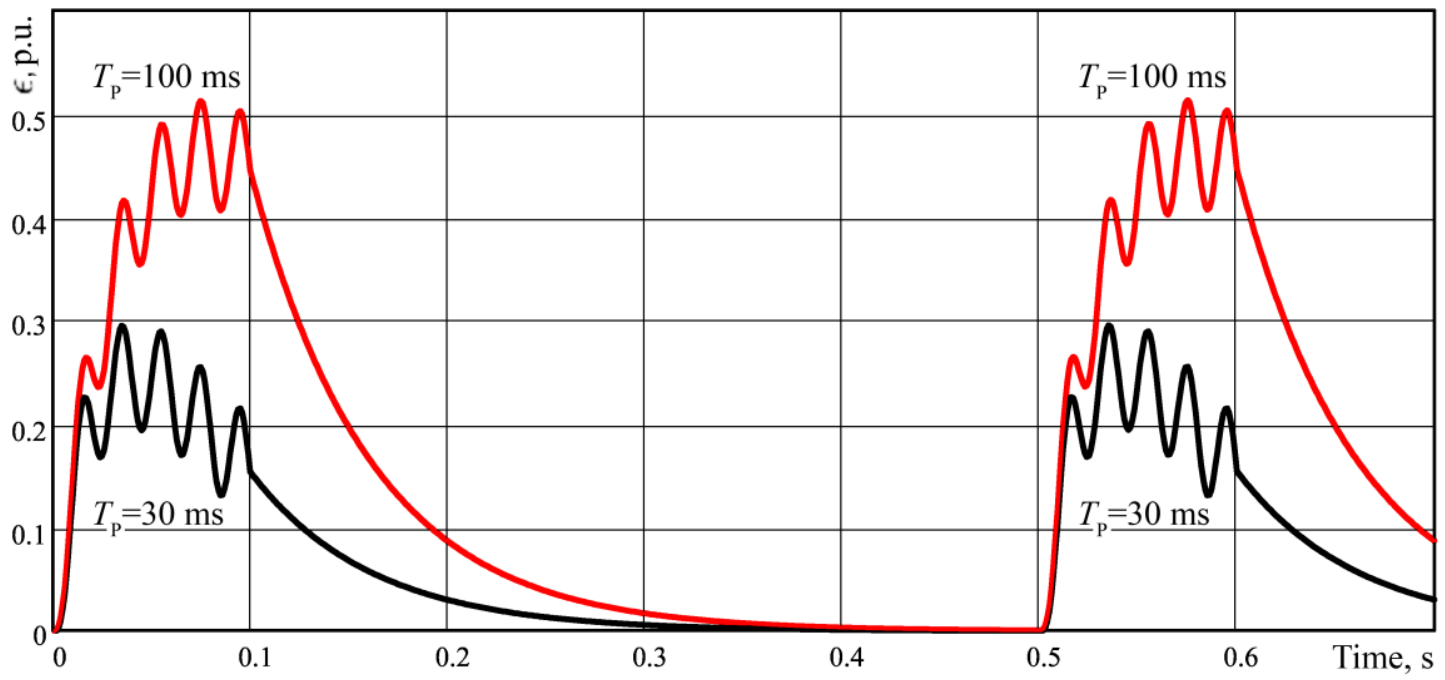

Fig. 3. Diagrams for Variation of Instantaneous Error in Automatic Reclosing Cycle for CT of TPZ accuracy class at $T_{p}=30 \mathrm{~ms} ; 100 \mathrm{~ms}$

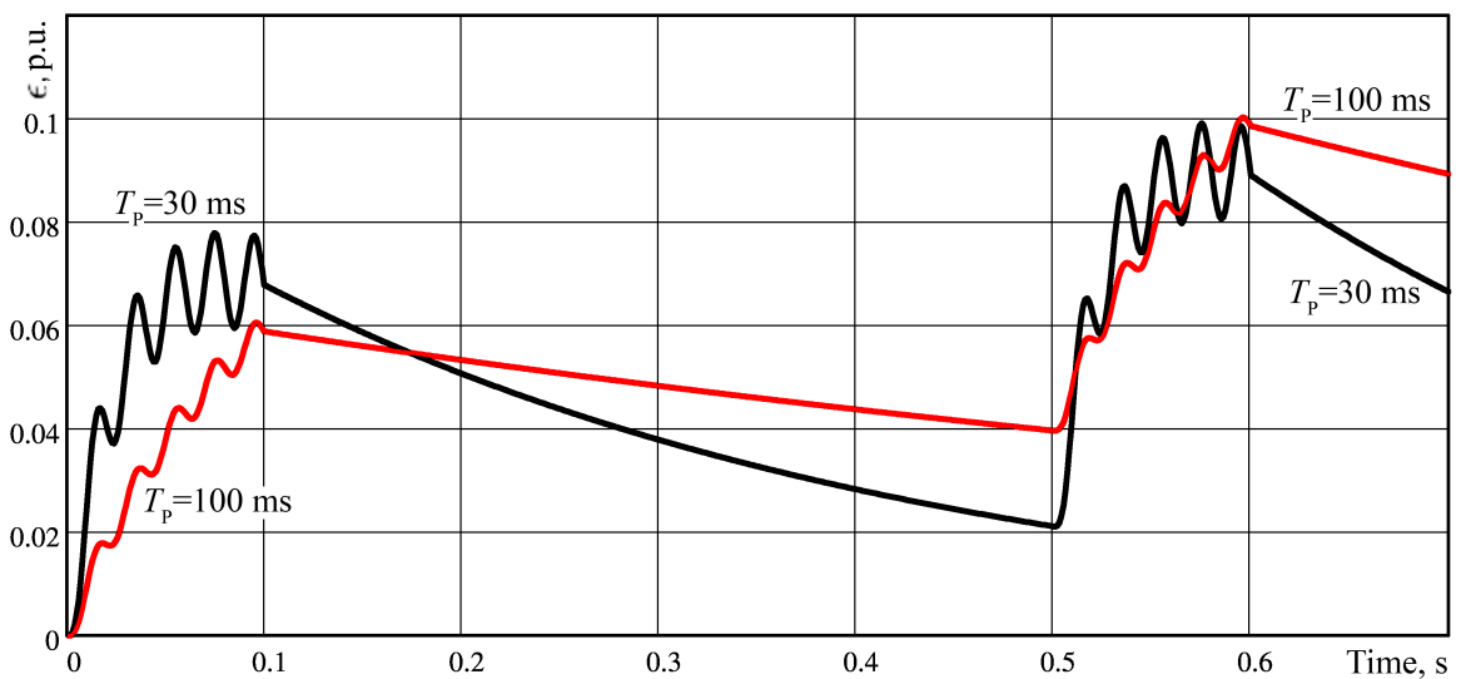

Fig. 4. Diagrams for Variation of Instantaneous Error in Automatic Reclosing Cycle for CT of TPY accuracy class at $T_{p}=30 \mathrm{~ms} ; 100 \mathrm{~ms}$.

\section{Conclusion}

Particular features of phenomena inside gapped core current transformers are examined. A procedure is proposed that allows for evaluating the excitation limiting e.m.f. and overall dimensions of the CT required for installation, based on known parameters of the transient condition in $\mathrm{CT}$ installation point and the specified CT error value. A possibility and a practicability to use the developed procedure for design activity (when selecting a CT) should be considered. On the experience of application, this procedure can be revised.

\section{References}

1. A. S. Sivkov, L. V. Shcheglov, G. A. Vedernikov, O. V. Petrova, Additional Parameters of Current
Transformers for Providing the Network Reliable Operation, Energoexpert, 3, 50 (2018)

2. V. S. Vorobiev, A. A. Degtyarev, S. L. Kuzhekov, V. V. Moskalenko, A. A. Navolochny, G. S. Nudelman, O. A. Onisova, A. I. Rasshcheplyaev, Functioning Investigation Results of the Relay Protection Devices in Transient Modes, Used in the Russian Federation, Accompanied by Current Transformers Saturation, RUM: Guiding Materials for Design and Operation of Electrical Networks. Scientific and Technical Review, 5, 22 (2018)

3. V. V. Afanasiev, N. M. Adoniev, V. M. Kibel et al, Current Transformers (1989)

4. PNST 283-2018. Instrument Transformers. Part 2. Requirements for Current Transformers (2018) 